\title{
On the Translation of a Treatment's Effect on Disease Progression Into an Effect on Overall Survival
}

\author{
Steven M. Snapinn* and Qi Jiang
}

\author{
Global Biostatistical Science, Amgen, Thousand Oaks, CA 91320, USA
}

\begin{abstract}
There are many examples of treatments for cancer that show a large and statistically significant improvement in progression-free survival (PFS) but fail to show a benefit in overall survival (OS). One recent example that has received considerable attention involves bevacizumab (Avastin) for the treatment of breast cancer. While it seems logical that slowing the rate of progression of a fatal disease would translate into an increase in survival, it is not clear what relative magnitudes of these two effects one should expect. One potential model for the translation of a benefit on disease progression into an OS benefit assumes that patients transition from a low-risk state (pre-progression) into a high-risk state (post-progression), and that the only impact of the treatment is to alter the rate of this transition. In this paper we describe this model and present quantitative results, using an assumption of constant hazards both preprogression and post-progression. We find that an effect on progression translates into an effect on survival of a smaller magnitude, and that two key factors influence that relationship: the magnitude of the difference between the hazard rate for death in the pre- and post-progression states, and the duration of follow-up.
\end{abstract}

Keywords: Oncology, Overall survival, Progression-free survival, Restricted mean, Bevacizumab.

\section{INTRODUCTION}

There is an ongoing debate over the usefulness of disease progression as an endpoint in clinical trials of treatments for patients with cancer (e.g., [1]). One view is that disease progression is a clinically meaningful event even if the treatment has no effect on how long the patient survives or how well the patient feels or functions. However, that is not universally accepted, particularly in situations where disease progression is assessed by radiology and would be otherwise unnoticeable to the patient. Another view, therefore, is that disease progression is only important to the extent that it predicts the patient's expected survival or quality of life. For this reason, researchers have attempted to establish the disease progression as a surrogate for survival for various types of cancer, including prostate [2], breast [3], colorectal [4], and renal [5]. However, even in those situations where disease progression has been established as a surrogate for overall survival, it may not be clear what magnitude of benefit on survival to expect from a given benefit on disease progression. That is the issue addressed by this paper.

This issue was highlighted recently by the debate over the use of bevacizumab for the treatment of breast cancer [6]. While bevacizumab was shown to have a large effect on progression-free survival (an endpoint similar to disease progression, except that deaths that occur in subjects who have not progressed are considered to be endpoints), clinical trials did not

*Address correspondence to this author at Global Biostatistical Science, Amgen, 24-2-C, Thousand Oaks, CA 91320, USA; Tel: 805-313-4368; Fax: 805-375-1297; E-mail: ssnapinn@amgen.com demonstrate a benefit on survival, leading the US Food \& Drug Administration to withdraw the indication for bevacizumab's use in patients with breast cancer.

Chen and Sun [7] and Fleming, Rothmann and Lu [8] addressed the issue of the magnitude of benefit on disease progression that should be required for drug approval. Tang et al. examined the influence of survival post-progression on the translation of an effect on progression-free survival into an effect on overall survival in the setting of renal cell carcinoma [9]. Fleischer, Gaschler-Markefski and Bluhmki [10] examined an exponential model for the relationship between progression-free survival and overall survival, and derived formulae for the correlation between the two values, and other authors have addressed the issue of the relationship between progression-free survival and overall survival [11-13]. In particular, Dejardin, Lesaffre and Verbeke [14] described a multistate model for the association between disease progression and overall survival. According to this model, a patient begins in an initial pre-progression state, and can transition directly into either a postprogression state or a death state, each with its own rate parameter. In addition, patients in the postprogression state can transition into the death state, with a separate rate parameter. This is the model that we use to quantify the magnitude of effect on overall survival that one should expect for a given effect on disease progression.

\section{MODEL FOR THE RELATIONSHIP BETWEEN DISEASE PROGRESSION AND SURVIVAL}

Following the multi-state model described by Dejardin, Lesaffre and Verbeke [14], patients are 
assumed to be in one of two states, either preprogression or post-progression, and each state has a separate hazard function describing the risk of death over time. Although it is recognized that this may not be a realistic assumption in many cases, for simplicity we consider in this paper a simple model in which both hazard functions are constant over time. We denote the pre-progression hazard rate for death as $\mathrm{y}_{0}$ and the post-progression hazard rate for death as $\mathrm{Y}_{1}$. We also assume that patients progress from the preprogression state to the post-progression state with a constant hazard rate for progression, $y_{p}$. Finally, we assume that the impact of the treatment is to alter the rate of progression by the factor $\lambda$; therefore, the hazard rate for progression in the treated group is $\lambda \gamma_{p}$. We refer to $\lambda$ as the progression hazard ratio.

Under this model, the density function for death in the control group can be obtained by integration over the distribution of time-to-progression. It is convenient to consider separately progression prior to death and death prior to progression. Therefore,

\section{$f_{c}(t)$}

$=\int_{0}^{t} \operatorname{Pr}\{$ progress at time $u\} \operatorname{Pr}\{$ die at time $t \mid$ progress at time $u\} d u$ $+\int_{t}^{\infty} \operatorname{Pr}\{$ progress at time $u\} \operatorname{Pr}\{$ die at time $t \mid$ progress at time $u\} d u$

or

$$
f_{c}(t)=\int_{0}^{t} \gamma_{p} e^{-\gamma_{p} u} \cdot e^{-\gamma_{0} u} \cdot \gamma_{1} e^{-1(t-u)} d u+\int_{t}^{\infty} \gamma_{p} e^{-\gamma_{p} u} \cdot \gamma_{0} e^{-\gamma_{0} t} d u
$$

or

$$
f_{c}(t)=\left(\gamma_{0}-\frac{\gamma_{1} \gamma_{p}}{\gamma_{0}+\gamma_{p}-\gamma_{1}}\right) e^{-\left(\gamma_{0}+\gamma_{p}\right) t}+\frac{\gamma_{1} \gamma_{p}}{\gamma_{0}+\gamma_{p}-\gamma_{1}} e^{-\gamma_{1} t} .
$$

Similarly, the density function for death in the experimental (treated) group is

$$
f_{e}(t)=\left(\gamma_{0}-\frac{\gamma_{1} \gamma_{p}}{\gamma_{0}+\lambda \gamma_{p}-\gamma_{1}}\right) e^{-\left(\gamma_{0}+\lambda \gamma_{p}\right) t}+\frac{\gamma_{1} \lambda \gamma_{p}}{\gamma_{0}+\lambda \gamma_{p}-\gamma_{1}} e^{-\gamma_{1} t}
$$

Note that when $\gamma_{0}+\gamma_{p}=\gamma_{1}, f_{c}(t)$ reduces to $\left(\gamma_{0}+\gamma_{1} \gamma_{p} t\right) e^{-\gamma_{1} t}$, and when $\gamma_{0}+\lambda \gamma_{p}=\gamma_{1}, f_{e}(t)$ reduces to $\left(\gamma_{0}+\gamma_{1} \lambda \gamma_{p} t\right) e^{-\gamma_{1} t}$.

The survival functions and hazard functions for the two treatment groups can be obtained from these density functions, as follows:

$$
\begin{aligned}
& S_{c}(t)=\left(\frac{\gamma_{0}}{\gamma_{0}+\gamma_{p}}-\frac{\gamma_{1} \gamma_{p}}{\left(\gamma_{0}+\gamma_{p}\right)\left(\gamma_{0}+\gamma_{p}-\gamma_{1}\right)}\right) e^{-\left(\gamma_{0}+\gamma_{p}\right) t} \\
& +\frac{\gamma_{p}}{\gamma_{0}+\gamma_{p}-\gamma_{1}} e^{-\gamma_{1} t} \\
& S_{e}(t)=\left(\frac{\gamma_{0}}{\gamma_{0}+\lambda \gamma_{p}}-\frac{\gamma_{1} \lambda \gamma_{p}}{\left(\gamma_{0}+\lambda \gamma_{p}\right)\left(\gamma_{0}+\lambda \gamma_{p}-\gamma_{1}\right)}\right) e^{-\left(\gamma_{0}+\lambda \gamma_{p}\right) t} \\
& \quad+\frac{\lambda \gamma_{p}}{\gamma_{0}+\lambda \gamma_{p}-\gamma_{1}} e^{-\gamma_{1} t} \\
& h_{c}(t)=\frac{\left[\gamma_{0}\left(\gamma_{0}+\gamma_{p}-\gamma_{1}\right)-\gamma_{1} \gamma_{p}\right] e^{-\left(\gamma_{0}+\gamma_{p}\right) t}+\gamma_{1} \gamma_{p} e^{-\gamma_{0} t}}{\frac{\gamma_{0}\left(\gamma_{0}+\gamma_{p}-\gamma_{1}\right)-\gamma_{1} \gamma_{p}}{\gamma_{0}+\gamma_{p}} e^{-\left(\gamma_{0}+\gamma_{p}\right) t}+\gamma_{p} e^{-\gamma_{1} t}} \\
& h_{e}(t)= \\
& \frac{\left[\gamma_{0}\left(\gamma_{0}+\lambda \gamma_{p}-\gamma_{1}\right)-\gamma_{1} \lambda \gamma_{p}\right] e^{-\left(\gamma_{0}+\lambda \gamma_{p}\right) t}+\gamma_{1} \lambda \gamma_{p} e^{-\gamma_{0} t}}{\gamma_{0}\left(\gamma_{0}+\lambda \gamma_{p}-\gamma_{1}\right)-\gamma_{1} \lambda \gamma_{p}} e^{-\left(\gamma_{0}+\lambda \gamma_{p}\right) t}+\lambda \gamma_{p} e^{-\gamma_{1} t}
\end{aligned}
$$

Note that at time $t=0$ the hazard rate in each treatment group is $\gamma_{0}$ and the hazard ratio is 1 . As $\mathrm{t} \rightarrow \infty$, the hazard rates depend on the values of $\gamma_{0}$, $\gamma_{1}, \gamma_{p}$ and $\lambda:$ In the control group the hazard rate approaches $\min \left\{\left(\gamma_{0}+\gamma_{p}\right), \gamma_{1}\right\}$, while in the treated group the hazard rate approaches $\min \left\{\left(\gamma_{0}+\lambda \gamma_{p}\right), \gamma_{1}\right\}$. Therefore, when $\gamma_{1}>\left(\gamma_{0}+\gamma_{p}\right)$, as $\mathrm{t} \rightarrow \infty$ the hazard ratio approaches $\frac{\left(\gamma_{0}+\lambda \gamma_{p}\right)}{\left(\gamma_{0}+\gamma_{p}\right)}$, while when $\gamma_{1}<\left(\gamma_{0}+\lambda \gamma_{p}\right)$, as $\mathrm{t} \rightarrow \infty$ the hazard ratio approaches 1 .

The mean survival time is obtained by integrating the survival function from time 0 to $\infty$, as follows:

$$
\begin{aligned}
& m_{c}=\frac{\gamma_{0} \gamma_{1}+\gamma_{p}\left(\gamma_{0}+\gamma_{p}+\gamma_{1}\right)}{\gamma_{1}\left(\gamma_{0}+\gamma_{p}\right)^{2}} \\
& m_{e}=\frac{\gamma_{0} \gamma_{1}+\lambda \gamma_{p}\left(\gamma_{0}+\lambda \gamma_{p}+\gamma_{1}\right)}{\gamma_{1}\left(\gamma_{0}+\lambda \gamma_{p}\right)^{2}} .
\end{aligned}
$$

Similarly, the restricted mean functions can be obtained by integrating the survival functions from time 0 to $t$ :

$$
\begin{aligned}
& m_{c}(t)=m_{c}-\frac{\gamma_{0}\left(\gamma_{0}+\gamma_{p}+\gamma_{1}\right)-\gamma_{1} \gamma_{p}}{\left(\gamma_{0}+\gamma_{p}\right)^{2}\left(\gamma_{0}+\gamma_{p}-\gamma_{1}\right)} e^{-\left(\gamma_{0}+\gamma_{p}\right) t} \\
& -\frac{\gamma_{p}}{\gamma_{1}\left(\gamma_{0}+\gamma_{p}-\gamma_{1}\right)} e^{-\gamma_{1} t}
\end{aligned}
$$




$$
\begin{aligned}
& m_{e}(t)=m_{e}-\frac{\gamma_{0}\left(\gamma_{0}+\lambda \gamma_{p}+\gamma_{1}\right)-\gamma_{1} \lambda \gamma_{p}}{\left(\gamma_{0}+\lambda \gamma_{p}\right)^{2}\left(\gamma_{0}+\lambda \gamma_{p}-\gamma_{1}\right)} e^{-\left(\gamma_{0}+\lambda \gamma_{p}\right) t} \\
& -\frac{\lambda \gamma_{p}}{\gamma_{1}\left(\gamma_{0}+\lambda \gamma_{p}-\gamma_{1}\right)} e^{-\gamma_{1} t}
\end{aligned}
$$

While this is a proportional hazards model for disease progression, it is clearly not a proportional hazards model for survival.

\section{EVALUATION OF THE MODEL}

In this section we evaluate this model through a set of graphs. We focus on three cases: a base case in which the post-progression hazard rate for death is 10fold higher than the pre-progression rate $\left(\gamma_{0}=0.1, \gamma_{1}=1.0\right)$, a second case where there is a larger gap between the hazard rates in the pre- and post-progression states $\left(\gamma_{0}=0.1, \gamma_{1}=5.0\right)$, and a third case where the gap between the hazard rates is smaller $\left(\gamma_{0}=0.25, \gamma_{1}=0.5\right)$. These graphs all allow examination of the effect of treatment. In the case of the survival, hazard and restricted mean survival functions, this is done indirectly by presenting multiple curves for multiple values of $\gamma_{p}$ ranging from 0.2 to 2.0. So, for example, the curves for $\gamma_{p}=0.4$ and $\gamma_{p}=$ 0.8 illustrate the impact of a progression hazard ratio $(\lambda)$ of 0.5 . In the case of the hazard ratio and restricted mean ratio functions, this is done directly by presenting multiple curves for multiple values of $\lambda$, ranging from 0.2 to 0.9 .

Figure 1 illustrates the survival functions for this model in the three cases: the base case on top, followed respectively by the cases with a larger and smaller difference between the hazard rates in the preand post-progression states. The $x$-axis measures time in years. In the base case the effect of the treatment on survival (assessed by comparing survival curves with different values of $\gamma_{p}$ ) is moderately strong, but not as strong as the effect on progression. For example, compare the curves with $\gamma_{p}=0.4$ and $\gamma_{p}=0.8$, corresponding to a progression hazard ratio of 0.5 . Since the hazard rates for progression are constant, a hazard ratio of 0.5 corresponds to a doubling of the median time to progression. Median survival, on the other hand, is approximately 2.25 years with $\gamma_{p}=0.4$ and 1.65 years with $\gamma_{p}=0.8$, for a ratio of 1.36 . In the second case, in which the difference between the hazard rates in the pre- and post-progression states is larger than in the base case, the effect of the treatment on overall survival is larger, but still not as large as its effect on progression. Finally, in the third case, in which the difference between the hazard rates in the pre-and post-progression states is smaller than in the base case, the treatment appears to have little effect on overall survival, regardless of the magnitude of its effect on progression.

Figure 2 illustrates the hazard functions in the same three cases. As described above, all hazard functions begin at $\gamma_{p}$ at time zero, and reach a plateau that depends on the values of $\gamma_{0}, \gamma_{1}, \gamma_{p}$ and $\lambda$. In the base case the plateau is at 0.3 for $\gamma_{p}=0.2,0.5$ for $\gamma_{p}$ $=0.4,0.9$ for $\gamma_{p}=0.8$, and 1 for $\gamma_{p}=1.2$ and 2.0. Note that the values of the plateaus indicate a smaller treatment effect on survival than on progression. For
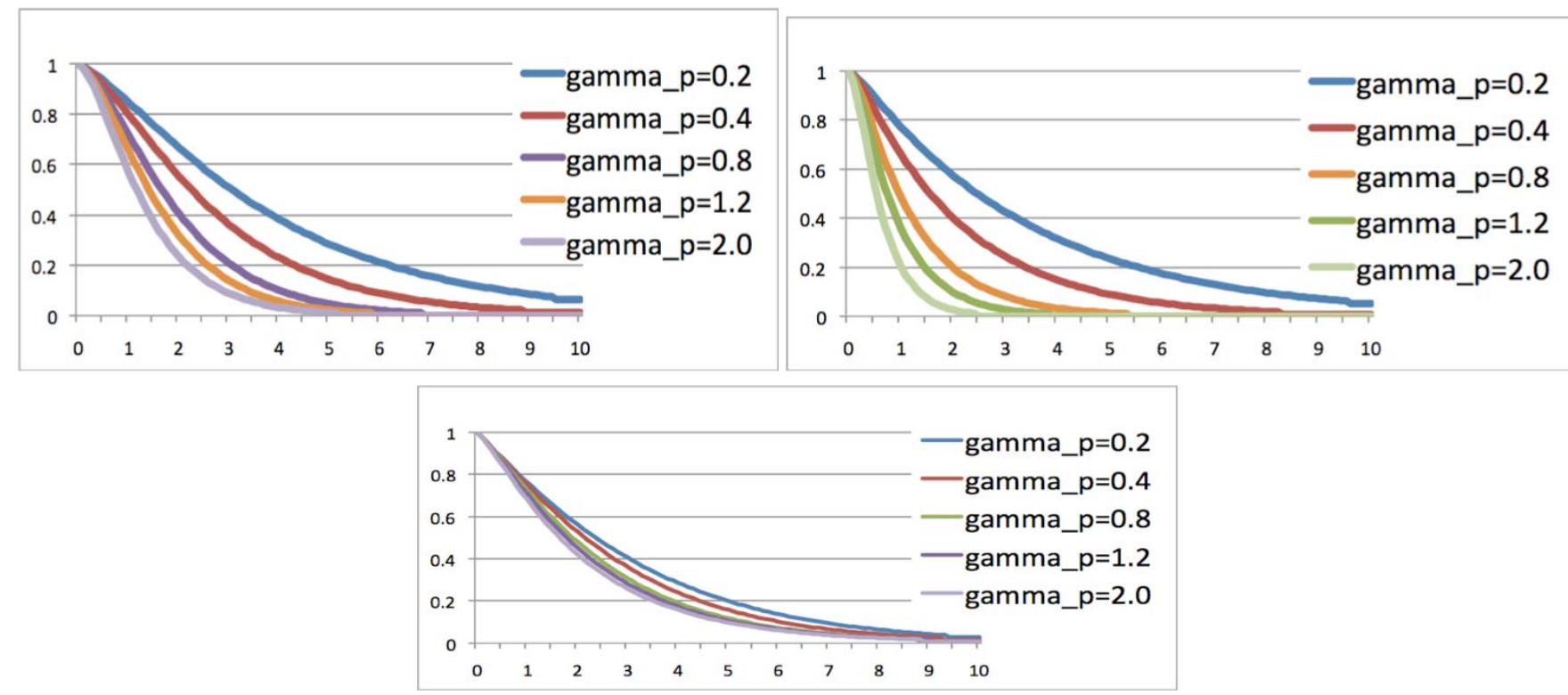

Figure 1: Survival functions in the base case (left) $\left(\gamma_{0}=0.1, \gamma_{1}=1.0\right)$, the second case (right) $\left(\gamma_{0}=0.1, \gamma_{1}=5.0\right)$, and the third case $\left(\gamma_{0}=0.25, \gamma_{1}=0.5\right)$, for various values of $\gamma_{p}$ ranging from 0.2 to 2.0 . 

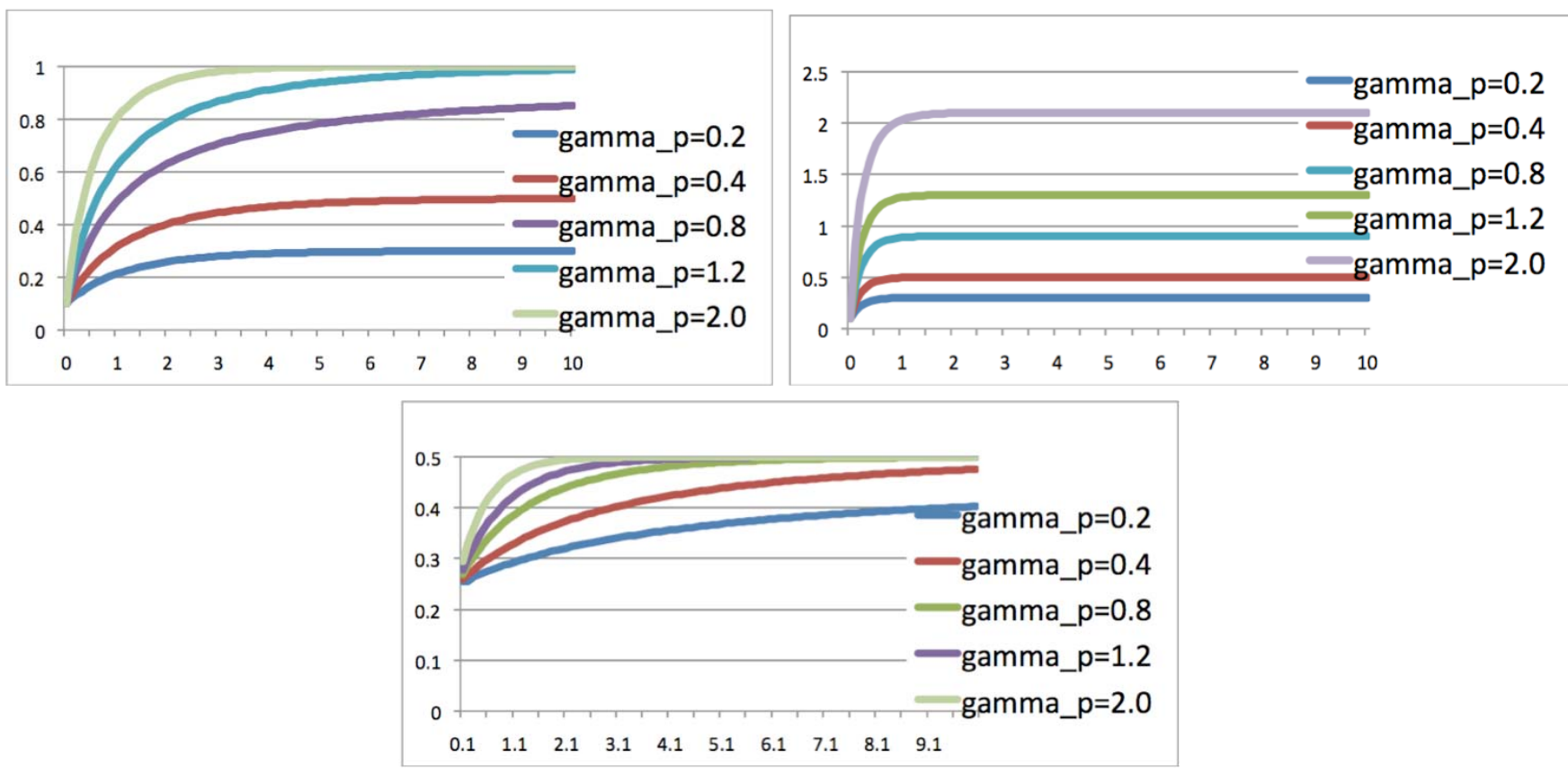

Figure 2: Hazard functions in the base case (left) $\left(\gamma_{0}=0.1, \gamma_{1}=1.0\right)$, the second case (right) $\left(\gamma_{0}=0.1, \gamma_{1}=5.0\right)$, and the third case $\left(\gamma_{0}=0.25, \gamma_{1}=0.5\right)$, for various values of $\gamma_{p}$ ranging from 0.2 to 2.0 .

example, again comparing the curves with $\gamma_{p}=0.4$ and $\gamma_{p}=0.8$, which correspond to a progression hazard ratio of 0.5 , the ratio of plateaus is 0.56 . Again, in the second case the effect of treatment on survival seems somewhat larger (but still not as large as its effect on progression) and in the third case the effect of treatment on survival seems relatively small.

The effect of the treatment on the hazard ratio, examined as a function over time, is illustrated in Figure 3. Unlike Figures 1 and 2, this figure evaluates a single value of $\gamma_{p}(0.5)$ and values of $\lambda$ ranging from 0.2 to 0.9 . This figure shows that the value of the hazard ratio at any point in time is always greater than the value of $\lambda$ (i.e., closer to the null), and sometimes much greater (especially in the third case).

Figures 4 and $\mathbf{5}$ illustrate the impact of the treatment on mean survival. Figure 4 illustrates the restricted mean survival over time, and Figure 5 illustrates the ratio of the restricted means. Although the hazard rates for survival are not constant over time, the plateau of the mean ratio curve can be thought of as an approximation to a hazard ratio for survival. Therefore, this could be considered to be the best measure of the overall, long-term effect of the treatment on survival. These figures illustrate that the magnitude of the effect of the treatment on survival is some fraction of its effect on progression, and that fraction increases as the difference between the hazard rates for death in the pre- and post-progression states increases. For example, with $\lambda=0.5$ the plateau of the mean ratio curve is approximately 0.72 in the base case, 0.62 in the second case, and 0.89 in the third case.

Figure 5 illustrates another key point: the restricted mean ratio decreases over time. Since the restricted mean ratio can be considered to approximate the effect on survival that would be observed in a clinical study, the apparent effect on survival will be smaller in a study of short duration, with lower mortality rates, than in a longer study in which the mortality rates are higher. For example, in the base case with $\gamma_{p}=0.5$ the survival rate in the control group would be expected to be about 0.78 at one year, 0.54 at two years, 0.11 at five years. The apparent treatment effect on survival in studies of those durations with $\lambda=0.5$ would be $0.97,0.93$ and 0.80 , respectively. This is despite the fact that the eventual effect on survival, if treatment were to continue, would be 0.72 . This is in contrast to a proportional hazards model for survival, in which case the effect of the treatment on survival would be constant, regardless of the duration of the study.

\section{DISCUSSION}

In this paper we evaluated a simple model for the effect of a cancer treatment on survival. In this model, patients are at either in a pre-progression state with a relatively low death rate, or in a post-progression state with a relatively high death rate; the treatment has no direct effect on survival, but indirectly improves survival by slowing the rate of progression. We assume that the hazard rates for death and progression are constant over time (i.e, exponential survival). Using this model, 

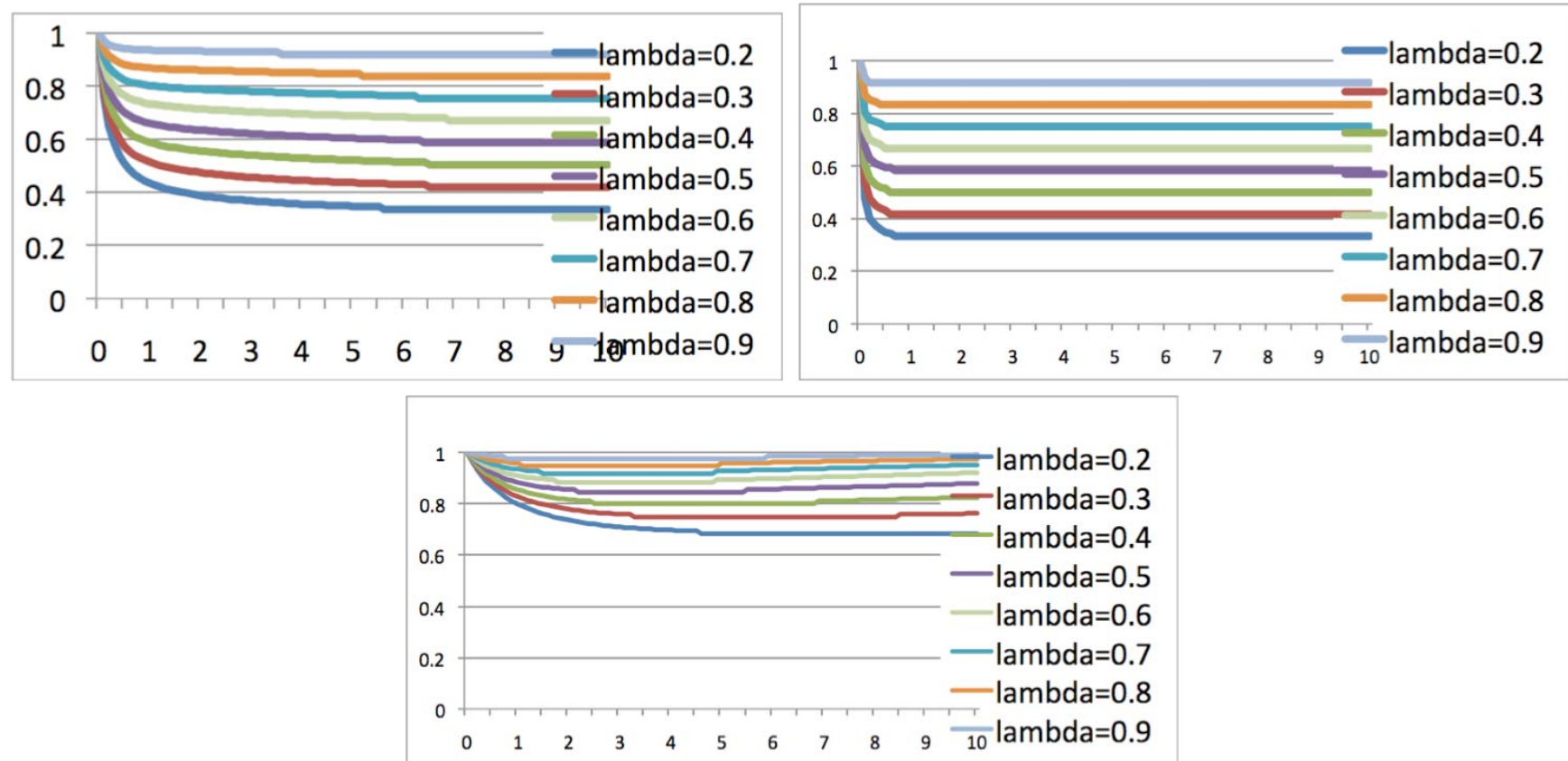

Figure 3: Hazard ratio functions in the base case (left) $\left(\gamma_{0}=0.1, \gamma_{1}=1.0\right)$, the second case (right) $\left(\gamma_{0}=0.1, \gamma_{1}=5.0\right)$, and the third case $\left(\gamma_{0}=0.25, \gamma_{1}=0.5\right)$, each with $\gamma_{p}=0.5$, for various values of $\lambda$ ranging from 0.2 to 0.9 .
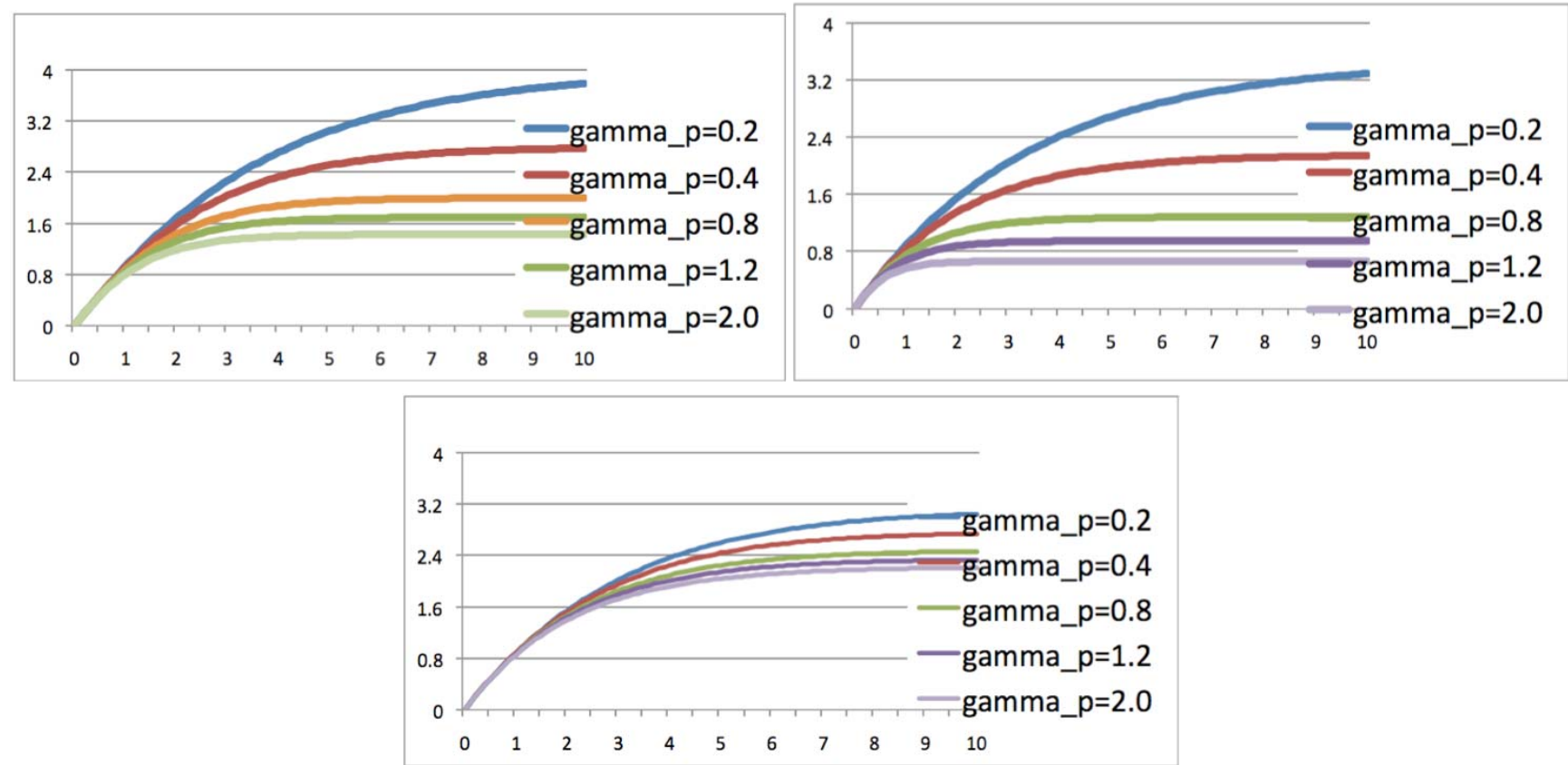

Figure 4: Restricted mean survival functions in the base case (left) $\left(\gamma_{0}=0.1, \gamma_{1}=1.0\right)$, the second case (right) $\left(\gamma_{0}=0.1, \gamma_{1}=\right.$ 5.0), and the third case $\left(\gamma_{0}=0.25, \gamma_{1}=0.5\right)$, for various values of $\gamma_{p}$ ranging from 0.2 to 2.0.

we explored the degree to which the effect of a treatment on progression is expected to translate into an effect on survival. We recognize that the model is quite simple and may not be very realistic. For that reason, we do not recommend focusing on precise quantitative results. However, we do believe that the qualitative results are informative, and provide insights that will carry over into more realistic situations.
One obvious result is that an effect on progression translates into an effect on survival of a smaller magnitude. This is not surprising, given that the treatment affects disease progression directly, but only affects survival indirectly. Two key factors influence that relationship. First, there is the magnitude of the difference between the hazard rate for death in the preand post-progression states. When the difference is 

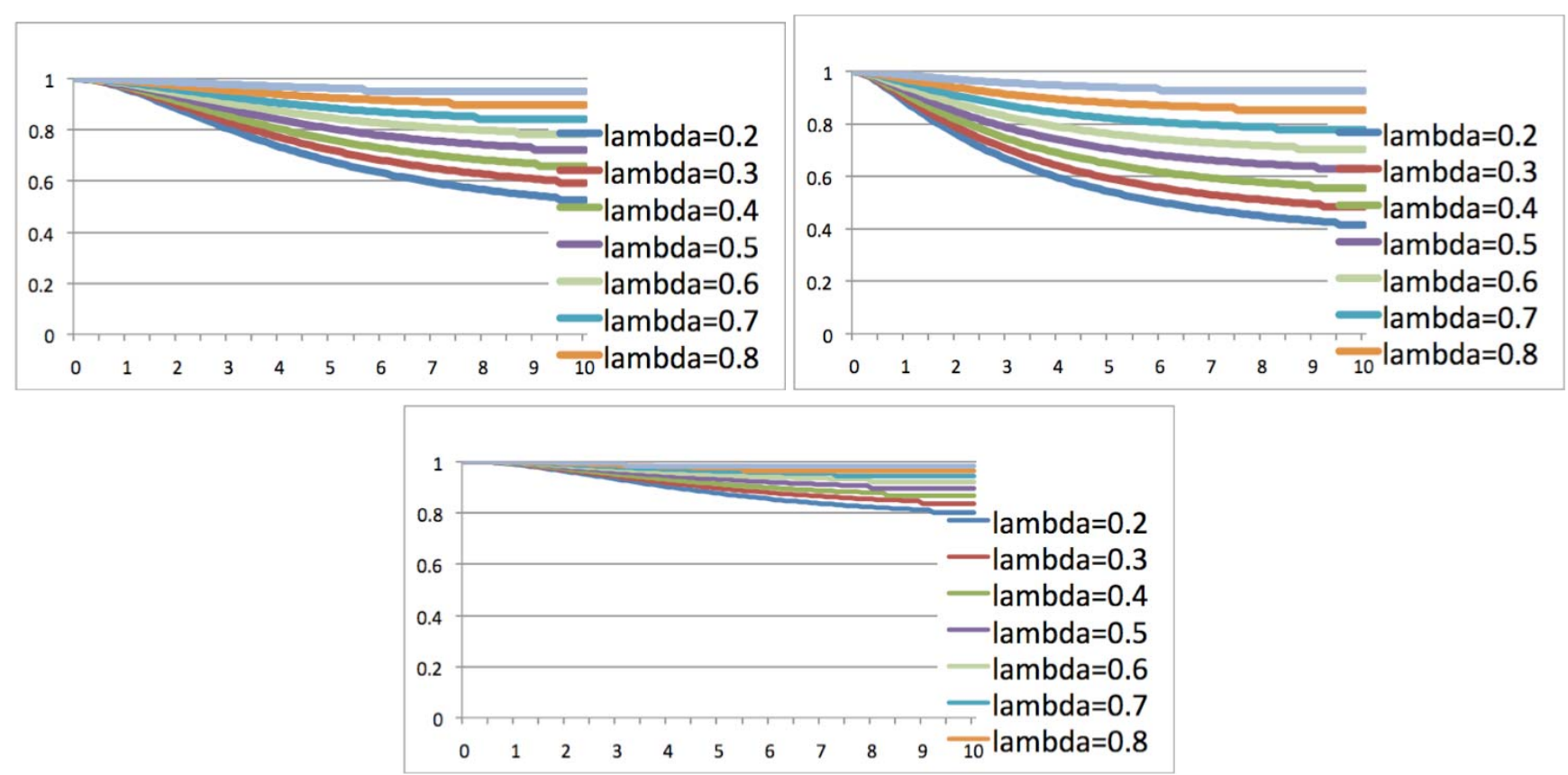

Figure 5: Restricted mean survival ratio functions in the base case (left) $\left(\gamma_{0}=0.1, \gamma_{1}=1.0\right)$, the second case (right) $\left(\gamma_{0}=\right.$ $\left.0.1, \gamma_{1}=5.0\right)$, and the third case $\left(\gamma_{0}=0.25, \gamma_{1}=0.5\right)$, each with $\gamma_{p}=0.5$, for various values of $\lambda$ ranging from 0.2 to 0.9 .

large the magnitude of the effect on survival can be nearly as great as the magnitude of the effect on progression, but when the difference is small the effect on survival will be small regardless of the magnitude of the effect on progression. In the extreme, if the preprogression hazard rate were zero, then an affect on progression would be equivalent to an effect on survival, and if the pre-progression hazard rate were equal to the post-progression hazard rate, then the treatment cannot influence survival.

The second factor is the duration of follow-up. Since the hazard ratio for survival is not proportional over time, we used the ratio of restricted mean survival over time as an approximation for the hazard ratio that would be calculated in a clinical trial. Using this measure, the apparent treatment effect on survival can be considerably smaller in a study of short duration than in a study of long duration. In one typical example, a $50 \%$ reduction in risk of progression would translate into approximately a $28 \%$ reduction in risk of death (as measured by the ratio of mean survival rates) if treatment continued for the remaining lifespan of all patients. However, if the study duration was only long enough such that $54 \%$ of the control patients were still alive, the apparent treatment effect on survival would translate into only an approximate $7 \%$ reduction in risk. This result could have important implications for the interpretation of clinical trial results when the duration of follow-up is relatively short.

\section{REFERENCES}

[1] Soria JC, Massard C, Le Chevalier T. Should progressionfree survival be the primary measure of efficacy for advanced NSCLC therapy? Annals of Oncology 2010; 21: 2324-2332. http://dx.doi.org/10.1093/annonc/mdq204

[2] Halabi S, Vogelzang NJ, Ou S-S, Owzar K, Archer L, Small EJ. Progression-free survival as a predictor of overall survival in men with castrate-resistant prostate cancer. Journal of Clinical Oncology 2009; 27: 2766-2771. http://dx.doi.org/10.1200/JCO.2008.18.9159

[3] Burzykowski T, Buyse M, Piccart-Gebhart MJ, Sledge G, Carmichael J, Lück, H-J, Mackey JR, Nabholtz J-M, Paridaens R, Biganzoli L, Jassem J, Bontenbal $M$, Bonneterre J, Chan S, Basaran A, Therasse P. Evaluation of tumor response, disease control, progression-free survival, and time to progression as potential surrogate endpoints in metastatic breast cancer. Journal of Clinical Oncology 2008; 26: 1987-1992.

http://dx.doi.org/10.1200/JC0.2007.10.8407

[4] Buyse M, Burzykowski T, Carroll K, Michiels S, Sargent DJ, Miller LL, Elfring GL, Pignon J-P, Piedbois P. Progressionfree survival is a surrogate for survival in advanced colorectal cancer. Journal of Clinical Oncology 2007; 25: 5218-5224. http://dx.doi.org/10.1200/JCO.2007.11.8836

[5] Halabi S, Rini B, Escudier B, Stadler WM, Small EJ. Progression-free survival as a surrogate endpoint of overall survival in patients with metastatic renal cell carcinoma. Cancer 2014; 120: 52-60. http://dx.doi.org/10.1002/cncr.28221

[6] Dienstmann R, Ades F, Saini KS, Metzger-Filho O. Benefit risk assessment of bevacizumab in the treatment of breast cancer. Drug Safety 2012; 35: 15-25.

http://dx.doi.org/10.2165/11595910-000000000-00000

[7] Chen C, Sun LZ. Quantification of PFS effect for accelerated approval of oncology drugs. Statistics in Biopharmaceutical Research 2011; 3: 434-444. http://dx.doi.org/10.1198/sbr.2011.09046 
[8] Fleming TR, Rothmann MD, Lu HL. Issues in using progression-free survival when evaluating oncology products. Journal of Clinical Oncology 2009; 27: 2874-2880. http://dx.doi.org/10.1200/JCO.2008.20.4107

[9] Tang Y, Bycott P, Akerborg O, Jonsson L, Negrier S, Chen C. Interpreting overall survival results when progression-free survival benefits exist in today's oncology landscape: a metastatic renal cell carcinoma case study. Cancer Management Research 2014; 6: 356-371.

[10] Fleischer F, Gaschler-Markefski B, Bluhmki E. A statistical model for the dependence between progression-free survival and overall survival. Statistics in Medicine 2009; 28: 26692686. http://dx.doi.org/10.1002/sim.3637

[11] Broglio KR, Berry DA. Detecting an overall survival benefit that is derived from progression-free survival. Journal of the National Cancer Institute 2009; 101: 1642-1649. http://dx.doi.org/10.1093/jnci/djp369
[12]

Redman MW, Goldman BH, LeBlanc, M, Schott, A, Baker $\mathrm{LH}$. Modeling the relationship between progression-free survival and overall survival: the phase II/III trial. Cancer Clinical Research 2013; 19: 2646-2656.

http://dx.doi.org/10.1158/1078-0432.CCR-12-2939

[13] Zhang L, Ko C-W, Tang S, Sridhara R. Relationship between progression-free survival and overall survival benefit: a simulation study. Therapeutic Innovation and Regulatory Science 2013; 47: 95-100. http://dx.doi.org/10.1177/0092861512459180

[14] Dejardin D, Lesaffre E, Verbeke G. Joint modeling of progression-free survival and death in advanced cancer clinical trials. Statistics in Medicine 2010; 29: 1724-1734. http://dx.doi.org/10.1002/sim.3918

(C) 2015 Snapinn and Jiang; Licensee Lifescience Global.

This is an open access article licensed under the terms of the Creative Commons Attribution Non-Commercial License (http://creativecommons.org/licenses/by-nc/3.0/) which permits unrestricted, non-commercial use, distribution and reproduction in any medium, provided the work is properly cited. 